\title{
Problems in implementing a good food and nutrition policy in Britain
}

\author{
W. P. T. JAMES \\ M.A., M.D., F.R.C.P. \\ Dunn Clinical Nutrition Centre, Addenbrooke's Hospital, \\ Trumpington Street, Cambridge CB2 1QE
}

\begin{abstract}
Summary
There is no co-ordinated food and nutrition policy in Britain, primarily because it is still not recognized that diet has an important role to play in the development of many diseases of affluence. The demand for absolute proof of the dietary component of disease on a population level ignores both the innate biological susceptibility in only a portion of the population to a particular nutrient as well as the increasing evidence from studies on non-human primates that diet plays a key role in several conditions including heart disease.

Government remains unsure of how to implement its own current recommendations and the traditional paternalistic and conservative approach delays action. Vested interests, seeking only short-term gains, are not confined to industrial concerns. Marketing Boards are allowed almost unfettered freedom to promote nutritionally undesirable eating habits. Health education in schools remains inadequate with most of the involved teachers still promoting incorrect concepts. The medical profession is still unwilling to take a preventive approach to disease with the hospital physician maintaining treatment programmes for obesity, diabetes and heart disease which have only marginal effects on a public health problem. General practitioners are better able to start practising preventive medicine but they need to be helped by community physicians who should begin on their main task of implementing policies for preventing disease rather than limiting themselves to their current preoccupation with systems analyses and operational research.
\end{abstract}

\section{Introduction}

The food policy in the United Kingdom necessarily reflects practical, economic and political considerations in addition to health and nutritional policies. Until recently the primary aim of Government has been to ensure that food is grown, processed, distributed and marketed in a safe manner. The emphasis has therefore been on standards of hygiene and toxicology. In addition, the Department of Health and Social Security (DHSS) encouraged the
Ministry of Agriculture to control food processing so that the nutritional adequacy of certain key items of the diet would not be compromised. The Department recommended, for example, that white bread should be supplemented with vitamins and minerals, that national dried milk should be withdrawn because of its inappropriate composition and suggested that the fortification of infant milks with vitamin $D$ should be reduced when it became apparent that the initial fortification programme had led not only to a rapid decline in the problem of rickets, but also to an increase in cases of hypercalcaemia. These specific nutritional approaches stem mainly from war-time food policies and in the light of modern knowledge some of the existing regulations are either no longer relevant to the prevention of disease or an inappropriate system for combating the problems encountered in Britain. Thus the iron which continues to be used in the fortification of white bread, despite its being unavailable for absorption, should be changed so that another absorbable iron compound is used. Alternatively, the fortification should be discontinued. Outmoded concepts continue to dominate much of the thinking on the application of nutritional and food policies to health since it is still considered that the main aim of Government should be to ensure that the needs of small groups within the population with problems of nutritional deficiency are met.

These views ignore the increasing evidence that there are a number of prevalent conditions which have an important nutritional component but which do not necessarily relate to a deficiency state in the classic nutritional manner. Recently there has been a substantial shift in policy at the DHSS and nutritional guidance has now been given to the general population as a way of 'promoting health'. These guidelines, presented in the DHSS report 'Eating for Health', suggest a number of changes in the pattern of food consumption of the average person in the U.K. As such, they represent a departure in thinking and a recognition that there is sufficient 
evidence already available to allow those nutritional experts who advise the DHSS Committee on Medical Aspects of Food Policy (COMA) to conclude that the secular changes in food consumption apparent in the U.K. since World War II, are inappropriate.

\section{Political considerations preventing an implementation of a nutritional policy}

These guidelines were preceded by the COMA Report on diet and heart disease (DHSS, 1974) which clearly set out the desirability of reducing fat consumption. Since this report, however, there seems to have been no further action to implement the recommendations of the Advisory Committee. Thus the DHSS does not appear to influence government thinking on a huge range of issues which affect consumption patterns. For example, there seems little evidence that the U.K. approach to EEC negotiations on the pricing of agricultural products and on the measures needed to deal with the skimmed milk or butter mountains have any features which relate to preventive medicine. A nutritional view should now be heard without of course it being automatically assumed by Community Physicians or Nutritionists that their views should be of overriding importance.

\section{Food labelling and the promotion of foods}

Other approaches to implementing the nutritional policies are also being ignored. To reduce fat intake a number of simple measures are needed. An intelligent person wishing to alter his food habits in the light of Government advice would find such a task difficult, particularly if he derived an appreciable proportion of his food intake from manufactured food products. The fat content of these products should be known and, since the DHSS advocates reducing the intake of saturated fatty acids, the fatty acid composition should also be marked on the label. Current systems for labelling foods are not only unhelpful but actually confusingoften defying the attempts at understanding of reasonably educated professionals.

It has often been argued that the labelling of foods will increase food costs but little evidence has been produced to back this claim and the major food manufacturers have sufficient quality control already involved in the manufacture of their foods to allow values for the fat content of food to be given. Assigning values for the type of fatty acid would, however, reduce the flexibility which the manufacturer now has for modifying the source of his ingredients and thus maintaining a low priced product. This is clearly a problem but current thinking now suggests that there will be increasing pressure to maintain the proportions of the saturated and polyunsaturated fatty acids in foods at a relatively constant level. This will mean, therefore, that it will become increasingly difficult for a manufacturer to substitute one oil, such as coconut, for another, e.g. corn oil. Nevertheless, the shift in public attitudes is such that the small increase in price required to produce a consistent product of known nutrient content would probably be acceptable. By the time this paper is published it will be clear that the Ministry of Agriculture's Advisory Committee, the Food Standards Committee, will have advocated the labelling of those foods for which distinct nutritional claims are made as part of the promotion of the product. This still means that manufactured foods such as sausages, pork pies, hamburgers, can have very varied fat contents without the customer knowing, and this is inadvisable. It is hoped that vegetable oils and table spreads will be labelled in the near future but there seem to be no valid reasons for exempting food products with a high fat content from food labelling. Public opinion is certainly moving in favour of there being more information and it is illogical to object on political grounds to the idea that government might influence people's eating habits, whilst making it difficult for the alternative freedom of choice to operate because the public cannot find out with eas the nutrient content of many commonly consume foods.

This seems so obvious that one could reasonable ask why food labelling has not been initiated sooner. Several reasons stand out. First, there is the traditional approach of the British Civil Service to social policy with an emphasis on an attempt to safeguard the community with appropriate standards rather than informing the public of possible dangers. The prevailing view seems to have been that the average citizen is unable to make appropriately balanced decisions and that Government should be conservative, awaiting good evidence before changing policy. Given this paternalistic attitude to the public's need to know about the content of food and the long-standing opposition of the main Consumer organizations to food labelling lest it raise food prices, it is not surprising that the food manufacturers saw no reason to increase the information provided on food labels. Nutritionists have also been singularly slow to advocate change, contenting themselves with only amateurish attempts at nutrition education.

The recent shift in nutritional thinking which began with the COMA Report on heart disease (DHSS, 1974), has been accompanied by a changed attitude to food labelling amongst consumers and their representative bodies. This has thrown food manufacturers on to the defensive, particularly when the new rigour of the 'Health Promoters' has been accompanied by the accusation that the food 
manufacturers are cynically disregarding the health of the community and are responsible for many of the prevalent diseases of affluence in the U.K. Some manufacturers have then responded by seeking to destroy the case of the Health Promoters rather than dispassionately evaluating the evidence. Indeed it could be argued that the food manufacturers have displayed the same inflexibility and unwillingness to 'cash-in' on the health connotation of some foods which seems to characterize other branches of industry.

This inflexibility of attitude also seems to affect the dairy industry and its reactions to those who advocate a reduction in saturated fatty acid intake. The dairy industry in Britain is unusual in having a daily door-step delivery of milk and much has been made of the social importance of this service for some groups, and particularly for the elderly. Yet attempts to reduce the fat content of the standard milk have been vigorously opposed so that a great opportunity to combine with Health Educators on an advertising campaign for the advantages of a 1-2\% fat milk have been lost. By ensuring that a legal minimum on ordinary milk is maintained at $3.75 \%$ fat, the Milk Marketing Board denies customers the freedom of choice and makes the U.K. unusual in E.E.C. law in not permitting a full range of milks. The present system also automatically incurs the criticism of modern nutritional and medical thinking and denies the general principle, evident in the publications of the Food Standards Committee, that food products can be modified so long as they fulfil various nutritional safeguards and do not mislead the public.

Further problems with implementing a coherent policy on food promotion by advertising also arise because the various independent Marketing Boards, e.g. the Egg and Potato Marketing Boards, do not have to comply with the restrictions on advertising which apply to other sectors of industry. Thus the emphasis on high fat products and their promotion in terms of health are quite wrong and the Potato Marketing Board's advertisements for the consumption of potatoes as chips rather than other forms of potato, ignores the conclusion of the COMA Report on heart disease (DHSS, 1974). Until these boards have to comply with the usual standards the public will continue to be misled.

There is also a need for the health profession to discuss the implications of the new ideas on health with industry. This process has already begun, with initiatives from the Centre for Agricultural Strategy (1978), but we still seem to be at the stage, noticeable in the U.S.A. many years ago, when the vested interests attempted to oppose change and initially failed to see the marketing opportunities which might develop as a result of these changes.

\section{Uncertainty in the scientific case for change}

The unwillingness to commit major organizations such as the National Health Service, the Department of Education and the Ministry of Agriculture, to work on implementing changes in food consumption stem not only from political and economic considerations, but also from a concern that the scientific case for change is insufficiently established. This is best illustrated by continuing controversy on the nutritional factors involved in the development of heart disease and on the possible benefits of a higher intake of polyunsaturated fatty acids. Some of the controversy stems from the very nature of the problem; most experts agree that absolute proof that lowering the intake of fat or increasing the consumption of polyunsaturated fatty acids does reduce the incidence of ischaemic heart disease is exceptionally difficult to gather and prohibitively expensive. Those who advocate that no advice be given to the public on changing their dietary habits then lamely suggest that 'more research' needs to be done; they fail, however, to advocate any lines of research which could lead to a conclusion which they would agree to judge as satisfactory. The sceptical experts are often physicians versed in the experimental approach of the metabolic ward, where studies are designed to deal with the quantitative effect of a single factor on the body's metabolism. When confronted with the interaction of a number of factors displayed by an epidemiological study, the metabolic physician concludes that the information is of negligible value in determining food policy unless an intervention study in the community clearly leads to marked changes in the incidence of the disease, and where the changes cannot be ascribed to any other factor. This approach is indeed ideal but in the absence of this information one has to resort in decisions on food policy to considering whether a link between, for example, saturated fat intakes and ischaemic heart disease has reasonably been made and whether the suggested change-to reduce fat intakes-would lead to more rather than fewer health problems. Given the prevalence of ischaemic heart disease, a policy of non-intervention can be considered a positive decision having major health implications if the problem might remit when the average intake of animal fats is reduced.

\section{Individual responsiveness to nutrients}

In most epidemiological studies it has been shown that within a population those with a high total serum cholesterol have a greater risk of developing heart disease. Reducing the serum cholesterol level artificially with drugs has now been shown to reduce the risk of heart disease, although iconoclasts point to the unchanged mortality rate in the treated group who had more gall stone formation and intestinal 
disease. This caveat is irrelevant to the principal question of whether lowering cholesterol reduces the incidence of ischaemic heart disease and only affects a policy decision on whether drugs should be used in the general population to bring about a reduction in serum cholesterol.

It is well recognized that the relationship within a population between the saturated fat intake and the serum cholesterol is poor-this lack of correlation has often been taken incorrectly to mean that the two are not causally connected. This ignores the crucial importance of individual responsiveness to dietary change. Figure 1, drawn from data presented by Keys, Anderson and Grande (1959), shows the

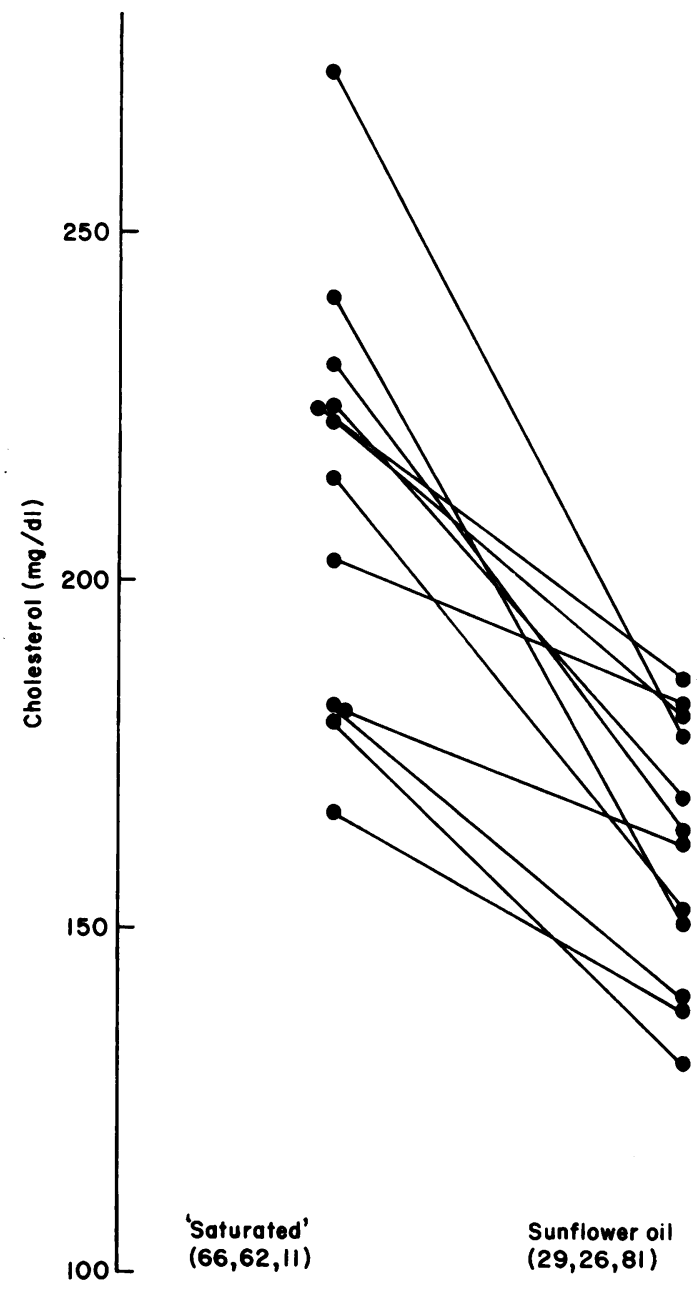

Fig. 1. The effect of ingesting first $137 \mathrm{~g}$ of saturated fat and then the same amount of sunflower oil on serum cholesterol in a group of young men. The numbers indicate the amount of saturated, monounsaturated and polyunsaturated fatty acids in each diet. Calculated from Keys, Anderson and Grande, 1959. 2-fold variability in the response of normal young men to a standard and constant diet containing $137 \mathrm{~g}$ of fat, and a predominance of saturated fatty acids. On transfer to an equivalent intake of polyunsaturated fatty acids, serum cholesterol values fell but there were differences in the response between individuals. Thus, the high responder to saturated fatty acids showed the greatest fall and in general the ranking of the individual values was maintained. This ranking effect is seen with a variety of biological processes including blood pressure and colonic function and has considerable health implications since it suggests that those individuals with values in the upper percentile range on a standard intake of saturated fatty acids, or for example salt or cereal fibre, are likely to show the greatest increase in serum cholesterol, BP or faecal output when the particular dietary stimulant is increased. The effect of individual responsiveness may therefore dominate the dietary effect in an epidemiological analysis unless one can find a population or populations with very varied intakes. This is illustrated in Fig. 2, taken from Keys and Kimura's (1970) analyses in 3 communities with very different fat intakes. Within each country there was no relationship between saturated fatty acid intake and serung cholesterol, but the relationship became apparent when the data from all 3 countries were combineof. One can then choose to emphasize the great import? ance of the genetic component to hypercholesterolaemia or recognize that those individuals with serum cholesterol values in the upper range are likely to respond best to a reduced saturated fatty acid intake.

In preventive medicine insufficient attention is being concentrated on those families whose members developed heart disease at a comparatively young age. This emphasis on families rather than on individuals, if combined with health education for the whole public, is preferable to any attempt to screen all children so that those in the upper range of serum cholesterol for their age could be identified. More specific risk factors are now being defined and children of parents with a low concentration of high density lipoprotein cholesterol and an increased risk of heart disease tend themselves to have a low concentration of HDL cholesterol (Van der Haar and Kromhout, 1978). A screening policy aimed at identifying these families would be unwise, since the technique is still relatively inaccurate and the approach is prohibitively expensive.

\section{Hypertension}

Hypertension is not only a well recognized risk factor for ischaemic heart disease, but has a synergistic effect on the development of heart disease when combined with obesity, hypercholesterolaemia or smoking (see Fig. 3). Furthermore, treating 
hypertension reduces the incidence of heart attacks and this evidence is now much more clear-cut than that for serum cholesterol. Much greater emphasis should now be made to identify hypertensive adults. Since $75 \%$ of all adults see their doctors once a year, a general policy by general practitioners of taking everybody's BP is overdue: if this were done by the clinic nurse, or even by a trained receptionist, this could represent a further genuine move by general practitioners into preventive medicine, and would begin to replace some of the medical work lost over the last 30 years to the hospitals.

Screening for hypertension could also be included in surveys of school health-a branch of the National Health Service which, although specifically geared to preventive medicine, seems to have lost its way. Percentile charts for children's BP are now available (Task Force, 1977) and it should be recognized that children who have BPs in the upper range for their age tend to retain their percentile position as they grow older and have a much greater likelihood of having hypertensive parents.

\section{Salt and high blood pressure}

Communities living in an environment where there is little opportunity to add salt to food not only have low BPs in early adulthood, but these persist at low levels throughout life. Primitive people ingest minute quantities of salt and yet survive in very hot tropical climates. The British adult, on average, consumes about $200 \mathrm{mmol} / \mathrm{day}$-about 20 times

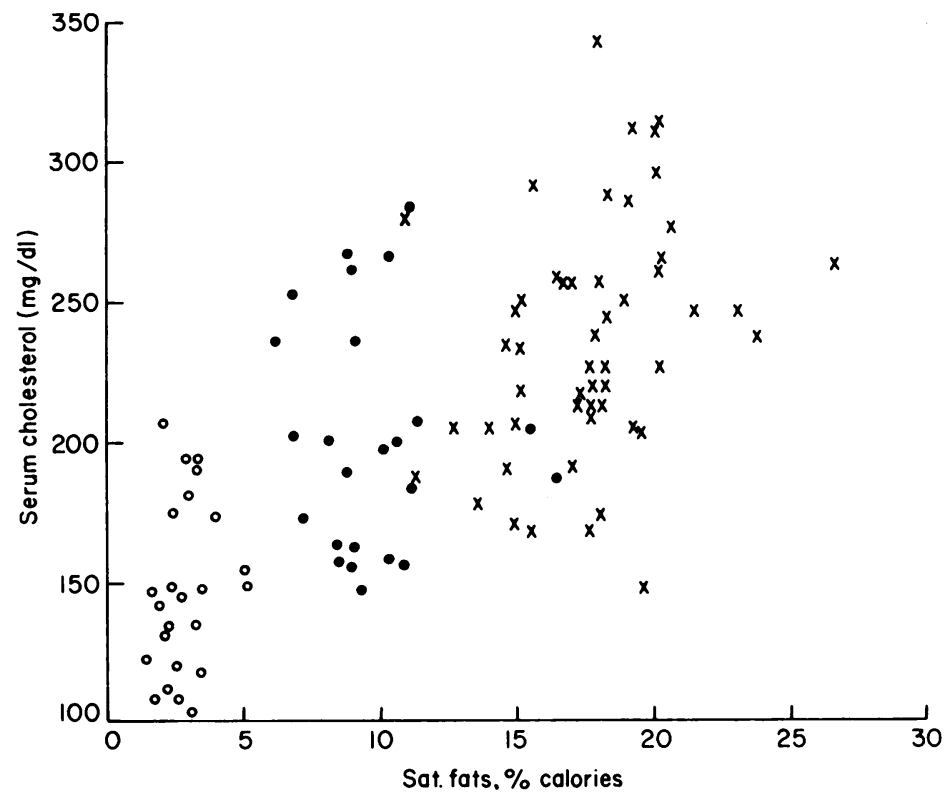

FIG. 2. The relationship between serum cholesterol and the proportion of dietary energy coming from saturated fat in rural Japanese (O) and the inhabitants of Crete (O), and Zutpehn ( $x$ ), in The Netherlands. Keys and Kimura, 1970. (With kind permission of the editor of American Journal of Clinical Nutrition.)

the minimum needs of the body. Salt is used to enhance the flavour of food but some primitive tribes in Africa use potassium chloride instead. Satisfactory salt substitutes are being developed which substitute potassium for sodium and these are on the market in Britain. As societies change their dietary habits and increase their salt intake, the prevalence of hypertension increases and deaths from strokes increase. The Japanese ingest vast amounts of salt, have a major public health problem of hypertension, and have the highest death rate from strokes in the world. As their food habits change, salt intakes are falling, as is the death rate from strokes.

Increasing the salt intake of many hypertensive patients leads to a rise in BP whereas this is not usually observed in normotensive subjects. These observations do not necessarily mean, however, that the hypertension is caused by excess salt intake - salt may merely exacerbate the condition. Nevertheless, animal studies by Dahl (1961) suggest that salt plays an important role in the generation of hypertension in some animals. By selective breeding he was able to develop a salt-resistant strain of rat which showed a progressive increase in BP as the salt content of the diet increased; in salt-insensitive animals there was no such relationship.

Recently, similar findings have been observed in man. Pietinen, Wong and Altschul (1979) selected adult subjects who were normotensive and divided them into 2 groups on the basis of whether their first 
degree relatives were diagnosed as being hypertensive. Three sequential $24-\mathrm{hr}$ urine samples were collected and related to the BP of the individuals. Figure 4 shows that there is a linear relationship between the urinary sodium output and the calculated mean BPs in those individuals with hypertensive relatives whereas no such relationship was found in people without a family history of hypertension. These findings may explain why some studies have failed to observe a relationship between 24-hr urine sodium output and BP: the salt-sensitive individuals form only a portion of the population so that the relationship between salt intake and hypertension may only become apparent in crosscultural comparisons. Studies conducted within a community tend to minimize the chance of finding this relationship because reliance is often placed on only a single BP reading and one collection of urine. Salt intake and BP are variable from day to day so that sequential measurements of urinary sodium and BPs are usually needed and even then the relationship will only be seen in a portion of the population.

In addition to the general relationship in saltsensitive subjects between salt intake and BP, new information demonstrates that hypertensive families have an abnormal physiological response to salt

(a)

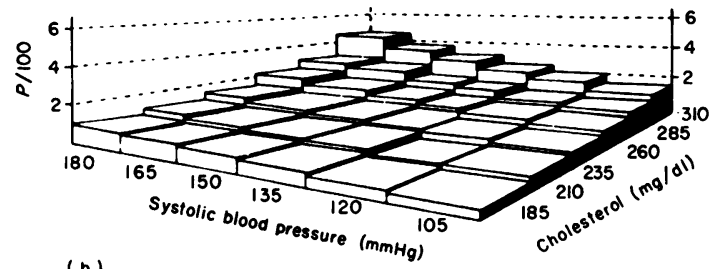

(b)

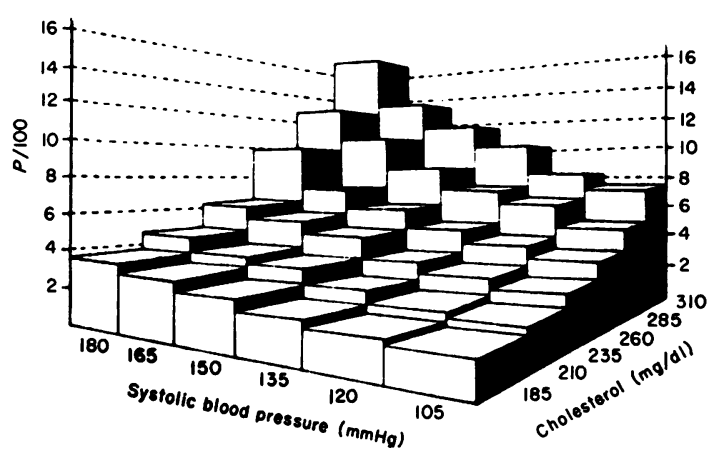

FIG. 3. The chance of a 35-year-old man having a heart attack within the next 6 years in relation to smoking, BP and the serum cholesterol concentration. (a) Non cigarette smoker, no LVH (left ventricular hypertrophy) or glucose intolerance. (b) Cigarette smoker, LVH and glucose intolerance present. (Taken from Borhani, 1977. With kind permission of the editor of American Journal of Cardiology.)

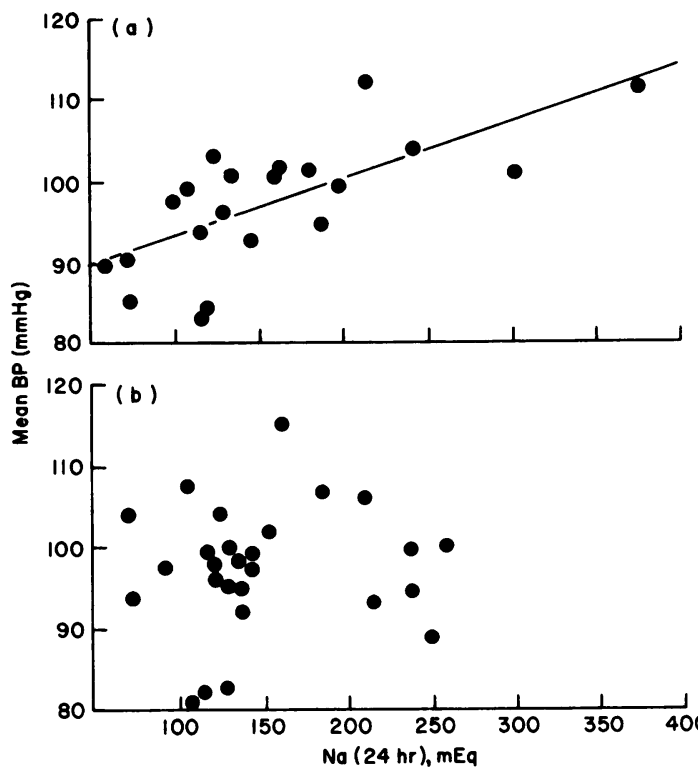

FIG. 4. The relationship between the output of sodium in the urine (equivalent to the dietary intake) and the BP of 2 groups of normal young adults. (a) Children of individuals with high BP. (b) Children without a family history of hypertension. (Figure redrawn from Pietinen et al., 1979. With kind permission of the editor of American Journal of Clinical Nutrition.)

loading which mimics that seen in salt-sensitive hypertensive rats. Normotensive sons of hypertensive individuals excrete sodium more rapidly in the normotensive phase than usual and have features in renal handling which indicate differences in proximal tubular functions (Wiggins, Basar and Slater, 1978; Bianchi et al., 1975). The same abnormality precedes the development of hypertension in saltsensitive rats. How this relates to the development of hypertension is unclear but this work and that of Pietinen et al. (1979) provide presumptive evidence for an abnormality in salt handling which precedes the development of hypertension. The habitual salt intake of individuals with a strong family history of hypertension does seem to relate to their BP levels $\subseteq$ even when these are still within the normal range.

On this basis it would not be unreasonable to advise hypertensive families to reduce their salt $N$ intake. There is also a clear need to assess the value of a community-based intervention programme to reduce salt intake and to monitor the effect on BP. $\omega$ This approach should yield more defined results than attempts to study fat intakes and heart $\varphi$ disease. Monitoring the BP of children and adults is $\overline{\mathbb{D}}$ a worth-while exercise if the appropriate standards $\stackrel{?}{?}$ are established and reliance is not solely placed on identifying individuals with diastolic BPs above $100 \mathrm{mmHg}$. 
Other problems in implementation: health education

The foregoing analysis was included to illustrate the way in which detailed consideration of epidemiological and metabolic data together can help to unravel those dietary factors which increase the risk of illness and explain the reasons for the resistance of some authorities to the claims made for the nutritional contribution to disease. Nevertheless, even when the DHSS is agreed on its policy, many major obstacles remain, particularly in the area of health education.

In the U.K. 'health education' is underfunded and has too general an approach to the national diet with the frequent use of relatively meaningless statements, e.g. on the importance of 'balanced diets' or the need to promote 'health'. A positive preventive medical approach based on specific recommendations for avoiding medical problems is unusual. Few health education programmes are ever assessed, so that improvements in educational techniques tend to come from experience, new ideas or 'hunches' and rarely from carefully planned health education research. The general practitioner has barely begun to practise health education except through the Infant Welfare Clinics, which have proved to be the most responsive to new ideas. Community physicians still seem more concerned with analysing health care systems than promoting effective preventive medicine.

In the educational service the Schools Council is reconsidering its approach to nutritional education and the Open University plans to include new ideas on nutrition in its courses. These developments are needed since the domestic science teacher in schools is herself often ignorant of current ideas and tends to rely on personal prejudice rather than facts to promote her ideas, many of which are incorrect. The teachers therefore need teaching, and greater flexibility needs to be shown in using the school curriculum to bring home to children the relevance of a life style without cigarettes, but with a more varied diet of a lower sugar and fat content and containing more dietary fibre. In school there is no reason why boys should be excluded from this education process. This educational process should include an improved rather than a deteriorating school meal service, since school catering follows nutritional guidelines in theory only.

The nutritionally most ignorant professional group is probably the medical profession. Doctors seem bored and irritated by non-specific general statements made by nutritionists, but simple practical suggestions can have an effect as shown by the recent study on advice against smoking in general practice (Russell et al., 1979). Key targets for improving the family's diet may well be the pregnant mother, who often claims ignorance on health matters and should be a prime target for establishing appropriate food habits in the young. Obesity in children can be prevented by appropriate education of the mother in pregnancy (Craddock, 1978). Hypertension, coronary artery disease, obesity and diabetes are all familial conditions so that effort could be made to institute particular care in these families.

Most sectors of our community have been at fault in resisting change and will need to be involved if we wish to implement dietary recommendations. Implementation must be undertaken in the knowledge that the current recommendations will change in the light of new knowledge and this concept that views will develop as understanding increases needs to be communicated to the public so that they do not become disenchanted by new ideas. This approach is preferable to a non-interventionist policy, which demands the type of proof for the benefit of dietary change which is unlikely to become available within the next decade.

\section{References}

Bianchi, G., Baer, P.G., Fox, U. \& Pagetti, D. (1975) Kidney function and blood pressure in a genetic type of hypertension. In: Sixth International Congress of Nephrology, Florence (Ed. Giovanetti, S.A.), pp. 274-283. Karger, Basel.

BorhANI, N.O. (1977) Primary prevention of coronary heart disease: a critique. American Journal of Cardiology, 40, 251.

Centre for Agricultural Strategy (1978) Food, Health and Farming: reports of panels on the implications for U.K. agriculture. CAS paper 7. (Ed by C. J. Robbins) Centre for Agricultural Strategy, Reading.

CRADDOCK, D. (1978) Obesity and its Management. 3rd edn. Churchill Livingstone,

DAHL, L.K. (1961) Effects of chronic excess salt feeding: induction of self-sustaining hypertension in rats. Journal of Experimental Medicine, 114, 231.

Department of Health and Social Security (COMA) (1974) Diet and Coronary Heart Disease. Report on Health and Social Subjects, 7. H.M. Stationery Office, London.

Department of Health AND Social Security (1978) Feeding for Health. H.M. Stationery Office, London.

Keys, A., Anderson, J.T. \& Grande, F. (1959) Serum cholesterol in man: diet fat and intrinsic responsiveness. Circulation, 19, 201.

KEYS, A. \& KIMURA, N. (1970) Diets of middle-aged farmers in Japan. American Journal of Clinical Nutrition, 23, 212.

Pietinen, P.I., Wong, O. \& Altschul, A.M. (1979) Electrolyte output, blood pressure and family history of hypertension. American Journal of Clinical Nutrition, 32, 997.

Russell, M.A.H., Wilson, C., TAYloR, C. \& BAKER, C.D. (1979) Effect of general practitioners' advice against smoking. British Medical Journal, 2, 231.

Task Force on Blood Pressure Control in Children (1977) National Heart Lung and Blood Institute and National High Blood Pressure Education Program. Pediatrics, 59, 797.

VAN DER HAAR, F. \& KROMHOUT, D. (1978) Food intake, nutritional anthropometry and blood chemical parameters in 3 selected Dutch schoolchildren populations. Communications Agricultural University, Wageningen, The Netherlands, No. 78-9.

Wiggins, R.C., Basar, I. \& Slater, J.D.H. (1978) Effect of arterial pressure and inheritance on the sodium excretory capacity of normal young men. Clinical Science and Molecular Medicine, 54, 639. 